\title{
Study on Artistic Function of Landian Yao's "Du Jie" Ceremony in Funing County of Wenshan of Yunnan Province
}

\author{
Jie Dong \\ Qujing Normal University \\ Qujing, China
}

\begin{abstract}
Landian Yao 's "Du Jie" ceremony in Funing County ,Wenshan, Yunnan Province, also known as "Guo Fa", it is the traditional ceremony of Yao nationality's men must accept moral education, social responsibility and rituals before they enter the society. Based on study the educational process of Liandianyao's "Du Jie" ceremony, this article analyzes its music types, including folk songs, instruments, dance and other forms, and try to analyze its social function, moral education function and artistic features from an aesthetic point of view.
\end{abstract}

Keywords-Landian Yao in Funing County; "Du Jie" ceremony; the artistic function; analysis

\section{INTRODUCTION}

Landian Yao People are good at singing and dancing, they have a long tradition of singing, their history, culture, production, life and other all activities mostly reflected through the music of the variety of religious ceremonies. Landian Yao's "Du Jie" ceremony still retains strong ancient Chinese religious color until now, for generations they are in nature worship, Gods worship and ancestor worship stage with rich forms and complex content. The mild character of Yao people got a good reputation among the neighboring fraternal peoples, which is inseparable with the "Du Jie" Education of Yao youth from an early age. The moral education of "Du Jie" provides a comprehensive guideline with the relationship between internal members of Yao groups, between the individual and the collective, between the Yao and other ethnic groups, in order to form a social norms system combines constraint and education in people's moral consciousness.

\section{Distribution Profile OF LANDIAN YAO PEOPLE IN Funing CoUnTy, WENSHAN, YunNAN PROVINCE}

According to the 2000 census, the population of Yao was 2.1374 million, they were distributed in Guangxi, Guangdong, Hunan, Guizhou and Yunnan five provinces. Among them, Yunnan Yao people live mainly in Hekou, Maguan, Jinping, Funing, Guangnan, Malipo, Qiubei, Yuanyang, Lvchun and Honghe counties. Funing County has the largest population of Yao people. Funing Yao people are divided into Landian Yao, Daban Yao and Shan Yao three branches. Yao is one of the six main nations in Funing County, ranked third in the county, they were located in Dongbo, Guichao, Muyang, Xinhua, Lida,
Huajia, Ayong and other 330 village groups of 77 village committees in 13 villages. Dongbo village is the village Yao people live up to the highest concentration and with the highest population. The language of Landian Yao and Daban Yao are very close so they can communicate with each other, and their customs also close so these two have closer relationship. Landian Yao called themselves "Jinmen"; Daban Yao called themself "You mian". Shan Yao called themselves "Ya", and they can dot communicate with other two language branches, so their customs are very different. From the order of move in time, Landian Yao is the earliest, Daban Yao followed, Shan Yao is the last. Yao was constantly move and change their residence, during the long historical process to fight with nature, they formed a rich and colorful ethnic culture and "industrious and brave, self-improvement" national spirit.

\section{LANDIAN YAO’S "DU JIE" CEREMONY IN FUNING COUNTY}

Landian Yao's "Du Jie" ceremony, also known as "Guo $\mathrm{Fa}$, which is the young man's rite of passage, encourage the man to get married and have children. In "Du Jie" ceremony, religious people are divided into "Shi gong" and "Dao gong" two categories, they are two different denominations. Each village has its own "Shi gong" and "Dao gong", on the occasion of "Du Jie" ceremony, they are the moderator of the village, but they can also invite "Shi gong" and "Dao gong" from other villages together to conduct religious ceremonies. Although "Du Jie" ceremony is a kind of etiquette, it contains a rich national culture.

\section{A. Beginning Stage}

Seven days before the ceremony, the Dujie person shall burn incense in the master's house, from that day he was known as the "Xin en", Du Jie person must obey the Master's arrangement, lying in bed during the day for ascetic meditation and not allowed to see the outside blue sky, he shall not eat meat, drink alcohol or kill livestock, when eat, only Master and his wife are allowed to fill the bowl, and the meal shall only be ginger, salt and sugar and rice. He can eat meat and playing outside only after the master finished the Shoudu process to "Xin en". 


\section{B. The Transfer Stage}

The middle five days are Shoudu process of Dujie person, it started the next day, the main home use wine and meat to invite their family ancestors and family Gods to the family feast, and ask them to testify and do not let the devil to make troubles. Then waves of deafening sound of gongs and drums from far and near came to the main house, outside, the house there is the Master wearing red vestments and "Shi gong" and "Dao gong" wearing vestments embroidered with moon and stars, god images, dragon images and hold yellow and green flags in their hands, they are introduced to the front gate of Tiantingfu which set by the main house. When entering the door, there will be a man on behalf of a family God from the main house to propose a toast to the masters, to express the greeting to Heaven Father, Heaven Father lead "Xin en" to enter the Tianfu Shengdian, "Du Jie" ceremony started in the serious atmosphere. The masters first plane the drum and report the family God, they read the elegiac address: "Respectly notice to family ancestors and family gods, today we played drums gong for family grandchild's " Du Jie ", hopefully he can be successfully ordained ......" until "Xin en" finished selecting Master, they played drum and gong, drink tea and offer wine and dishes, and then sang the epic of Yao - Panwang, Yuhuang, Dimu, Longwang, Jiuma Sanniang and other gods epic in order to ask them to protect "Xin en" and remove the disaster of "Xin en", bless "Xin en" a safe life.

Held the Dutian Jietiao "Wutai Shan" ceremony. Climb the Wutai Shan also known as the "Dushi Gongjie", Dutian Jietiao is the most important part of the whole "Du Jie" ceremony, it related to the success or failure of the entire ceremony. When climbing Wutai Shan, "Shi gong" and "Dao gong" opened a way in front, "Xin en" slowly towards the Wutai Shan outside village under the leadership of teachers. Posing a square table next to the Wutai Shan, "Shi gong" and "Dao gong" chanted the mantra, then point the wooden ladder with the God knife, teachers helped "Xin en" to climb up the wooden ladder to "Wutai Shan" step by step. "Xin en" crossed their legs under the guidance of teachers, sat straight can not look around or laugh, he looked very devout. Then the sound of gongs and drums was up, the Masters sang the Jing song, and opened a big net woven with canes above it, at the bottom of the net shaded with three quilts and three blankets wait for "Xin en" to born. "Xin en" clenched fingers inward tightly and keeps his knees with both hands under the teachers' guidance, huddle up in a heap and rolling down the "Wutai Shan" with his face up, finally falling into the net. If when the "Xin en" fell to the ground, his hands were separately then it is considered his "Du Jie" is failed, then his life will be looked down on others, but if when the maters opened the quilts and found out their hands still hold together on his knees, then the "Du Jie" was success.

\section{Completion Stage}

The last three days was the completion stage, "Du jie" person attentively listened to Master's admonition. The writing master will sealed the hour copies of Jieshu with Shigong seal, Daogong seal and Master seal, one copy handed to "Xin en" for collection, one copy handed to master in order to supervise the words and deeds of "Xin en" and not let him violate the Ten
Commandments, after burnt up the remaining two copies the "Du Jie" was finished.

\section{The Music TyPe of Landian YaO'S "Du JiE" Ceremony In Funing County}

\section{A. Folk Songs in "Du Jie" Ceremony}

Landian Yao people love to sing, Li Diaoyuan, a literati in Qing dynasty was said in his "Yuedong Biji": "Yao people like to sing, the women has various singing ways, echoed each other." They sing in the labor time, they sing at all kinds of ceremonies too. Many families will invite two female singers to sing the Dujie songs during the Dujie ceremony, the two female singers have to sing the Dujie songs day and night according to the scripture content of "Shi gong" and "Dao gong" chanted. Du Jie songs told the hardship of life and bitterness of the history migrations, also expressed people's yearning and pursuit for a better life. Yao's folk song monographs include "Panwang Dage", "Song of Butterflies", "Zanpanhu" and so on. Yao's folk music belongs to Chinese music system, it uses the complete pentatonic: 12356 .

"Zanpanhu" is a non-melodic accompaniment song, the song is composed by the five tones, belongs to ethnic Pentatonic. Lyrics to the effect is: "Tai Chi and Pangu create the world, they established the first year and become the emperor; emperors in heaven and create many things, they create sky and earth; Emperor has three hundred sixty eyes, Pangu has three hundred sixty incarnations; hands and feet become the trees on the mountains, form the body from the hands and feet. "'First, the lyrics convey they wish the emperors in heaven create more things, turned into gods to bless and bring benefit to the future of those Dujie persons. Secondly, from the structure of the song, "Zanpanhu" is a onepiece structure consisting of four phrases. In A phrase, start with a female leading voice, rhythm change and repeat to cause audience's attention and impress the audience, and then there is the drum to render the atmosphere, express a breathtaking picture; In B phrase there is more content, start with male voice: "Tai Chi and Pangu create the world... " then repeat with the female unison together and two-part chorus, thus to further highlight the theme of music and deepen the singer's thoughts. Then, male unison and female unison sing separately to explain the contribution of Pangu Emperor; The $\mathrm{C}$ phrase again with bass, played the "changing" role in the "opening, developing, changing and concluding" and further illustrates the importance of Pangu to "Xin en"; D phrase is summary and conclusion of the whole song.

\section{B. The Instruments in "Du Jie" Ceremony}

On "Du Jie" ceremony, the Dharma instruments of Jieshi are the "Dharma Drum" (commonly known as "Qizi Gu"), and "gong", "cymbal" three essential "Artifact".

"Dharma Drum" is the most important props of Landian Yao, it is the symbol of totem worship and ancestor faith of Landian Yao, it is sacred to Yao people, it encourage Yao People's spirit, ignite Yap people's hope, so it is used in many religious activities. In Yao "Du Jie" ceremony, the drum is the most important instrument of "Shigong" and "Daogong". 
Dharma Drum betoken the prophecy " promote Buddhist doctrine, make universal salvation" to people, when beat the drum ,it beat the drum to make spirit clarify and ascetic meditation, so that the heart of those "Dujie" person will get peace and calm.

"Gong" is the earliest musical instrument that the China's southwest minorities use, it is round with arc surface, normally is copper structure, with the features of low sound, loud and intense, long lingering. In folk it normally used to express a tense atmosphere. This minority traditional folk instruments, along with the inheritance and development of Chinese people of all nationalities, has already become a musical instrument which loved by the masses. And in a variety of activities and ceremonies which held by Yao, it also has become one of the indispensable instruments.

"Cymbals" in ancient called "Tong bo", "Tong pan", in folk called "Cha", it is widely used in various ethnic groups' folk dance, entertainment activities and campaign activities. It is a common percussion instrument with a long history and strong musical performance.

\section{The Dance in "Du Jie" Ceremony}

Due to historical reasons, for a long time Yao people were in the frequent migration non settled status, so their social and economic development is very uneven. And with geographical factors and enclosed living environment, in order to adapt external struggle and internal national unity, common national mentality led them to keep the "Du Jie" ceremony which is the traditional custom of national centripetal force completely spread until now. The dances in Landian Yao's "Du Jie" ceremony divided into "Wen Dance" and "Wu Dance".

First, "Shingong" leads his two disciples worship the Gods, plays "Wen dance", invites the gods out. First dance with 8 direction and flower movements, dance to the right front of the opposite side, then play up and down worship dance one time, then turn around and continue to dance with lying 8 direction to the left rear of opposite side, and play worship dance one time again. Subsequently, the same movements face to the right rear, left front, right side, left side each direction once, the eight directions shall dance three times. Each procedure invited God will dance in this route and each God often have to invite three times, dance three times. For different Gods the dances are different too. There are two dance forms of "Shigong" dance: one is stand dance, the whole body upright; one is the squat dance, the whole body is downward like squat; the dace steps are Tadou step“ First lift left foot, one tread one step, one front one after, one yin and one yang, the beginning and end synchronization, foot horizontal straight section like the Tshaped support each other, walking up like the split step ". The dance step of "Shigong"'s "Wen dance" is Tadou; and when play the dance they have to play all the eight directions, when dancing around with word direction, three of them no one can go wrong, one wrong will affect the overall situation. Thus, the dance step of "Wen dance" is elaborate and also very important.

Secondly, "Daogong" starts to play "Wu dance", it mainly includes Qianxian dance and Jianxian dance. Before the "Wu dance", "Daogong" will lead the "Dujie" person to worship his parents and Sanqing, Sanyuan and other Gods. Then under the accompaniment of percussion instruments, "Daogong" dancing while chanting the text. The dynamic characteristic of "Wu dance" is based on rush ahead and single foot hop, it does not have certain procedure, normally solo dance to coordinate with text. The dance movement is single, mainly pay attention to the fixed gesture, through atmosphere and expression to express certain mood, and play the role of killing the ghosts and evils. Then begin to send away the Gods, play the Xunchao dance, "Shigong" and "Daogong" walked under the accompaniment of percussion instruments, they use Tadou steps to circle around a few times, meaning to send back all the Gods. Next is start the meal and the whole "Du Jie" ceremony will be finished.

\section{THE ARTISTIC FUNCTION OF LANDIAN YAO’S"DU JIE" CEREMONY}

\section{A. Landian Yao's "Du Jie" Ceremony Has an Important Function of Moral Education}

Landian Yao's traditional morality is the history and culture of the nation accumulated in the long process of development and continues to this day, widely permeated in the spiritual cultures like myths and legends, folk tales, religious taboos, as well as the life practices like labor, customs, food, clothing, shelter and transportation. It influence and dominate people's thinking, attitude to life and lifestyle, their moral education has a long history. Landian Yao's "Du Jie" ceremony retains a strong color of ancient Chinese religion, they are still in nature worship, ancestor worship and multi-gods worship stage, it has rich forms and complex content. The mild character of Yao people got a good reputation among the neighboring fraternal peoples, which is inseparable with the "Du Jie" Education of Yao youth from an early age. The moral education of "Du Jie" provides a comprehensive guideline with the relationship between internal members of Yao groups, between the individual and the collective, between the Yao and other ethnic groups, in order to form a social norms system combines constraint and education in people's moral consciousness. "Du Jie" moral education function is good to train the positive qualities of pursuing progress and exploring for the young people, with the gentle way of doing things such like "If I am right, then I shall be modest; if I am wrong, I shall correct it; if I am not wrong, I shall keep it", effectively mediate the interethnic relations and relationship between other ethnic group, which won a good reputation from brotherhood ethnic groups. Therefore, the traditional education conducted by the form of "Du Jie" ceremony has the important influence and infiltration function to the formation of the nation's values and moral norm.

\section{B. The Social Function of Landian Yao's"Du Jie" Ceremony}

Music of "Du Jie" ceremony has strong ethnic characteristic, and ethnic music culture can cause emotional reactions of people who from the same nation. Yao people live together in a arduous environment for a long time, through the folk music passing they not only can understand each other, but also they can agree and stimulate their inner emotional resonance, thus resulting in a strong national cohesion, solidarity and national unity heart. During the Landian Yao's "Du Jie" ceremony all families help each other, in this time showed a strong national 
cohesion function, let Yao people influenced by the religious music moral from their young age and trained their strong sense of national identity. Thus, "Du Jie" can play to role of maintain the unity of Yao people, it is an important carrier of national cohesion.

\section{The Materialistic Aesthetic Perception of Landian Yao's"Du Jie" Ceremony}

Landian Yao People believes that folk song culture not only can express people's feelings, but also is a reproduction of the aesthetic perception of history things. Based on this materialistic aesthetic thinking, to actively guide the development and dissemination of the narrative songs, and thus has a major impact on music culture. The material carrier of dance is human ,human is a wonderful thing which full of spirituality and carried spirit and soul, and in order to get the gods bless, our ancestors had to use the silent body language of dance, fantasy they can communicate with the gods, so to presents the contact, exchange, shaking hands between soul and soul. In general, that is people through listening to singers singing and dancers dancing, to appearance the past things and situations together back to the audience's aesthetic perceptions. This aesthetic thinking of Yao people embodied in the aesthetic process of traditional narrative song events, which occurs in the depths of Yao people's hearts, with a long history.

\section{CONCLUSION}

Yao people in their production life created a variety of folk music, the lyrics and the melodies have a certain rhyme and the contents are simple and decency. Research on this traditional culture will contribute to its contemporary inheritance and promotion.

\section{REFERENCES}

[1] Deng Hua. The national collective memory construction in ceremonytake Landian Yao's "Du jie" ceremony in West six village,Dongbo,Funing County, Wenshan, Yunnan Province as an example [J] Study of Ethnic Education, 2012, (23): 218-223 .

[2] Wang Zhifen. Symbolic meaning interpretation of Yunnan Zhuang Pomao song book[C]. Central University for Nationalities, 2009.

[3] Wang Hongwei. Du Jie - mysterious rite of passage for man [J]Tourism, 2006, 74-79.

[4] Du Yaxiong. Chinese Ethnic Folk Music Overview [M] Shanghai: Shanghai Conservatory of Music Press, 2014: 481. 